\title{
Diffusion Simulation and Lifetime Calculation at RHIC
}

\author{
N. P. Abreu, W. Fischer, Y. Luo, G. Robert-Demolaize
}

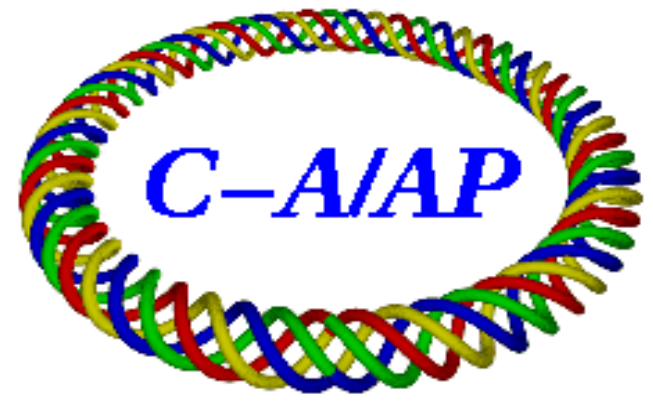

\section{Collider-Accelerator Department Brookhaven National Laboratory Upton, NY 11973}

Notice: This document has been authorized by employees of Brookhaven Science Associates, LLC under Contract No. DE-AC02-98CH10886 with the U.S. Department of Energy. The United States Government retains a nonexclusive, paid-up, irrevocable, world-wide license to publish or reproduce the published form of this document, or allow others to do so, for United States Government purposes. 
$\mathrm{C}-\mathrm{AD} / \mathrm{AP} / 346$

\title{
Diffusion Simulation and Lifetime Calculation at RHIC
}

\author{
N.P. Abreu, W. Fischer, Y. Luo and G. Robert-Demolaize
}

January 8, 2009

\begin{abstract}
The beam lifetime is an important parameter for any storage ring. For protons in RHIC it is dominated by the non-linear nature of the head-on collisions that causes the particles to diffuse outside the stable area in phase space. In this report we show results from diffusion simulation and lifetime calculation for the 2006 and 2008 polarized proton runs in RHIC.
\end{abstract}

\section{Introduction}

The lifetime measures the rate at which the circulating particles are lost due to a variety of effects like lattice non-linearities, intra-beam scattering and gas scattering, to give some examples. All those effects contribute to enhance the diffusion of the particles outside a stable area where they are lost after a certain number of turns. In this report we used the diffusion to estimate the lifetime of a polarized proton beam during a physics store, which is usually 5 hours long. The basic idea is that for protons in RHIC the main contribution to the diffusion are the head-on collisions at the two experiments, PHENIX (in IP8) and STAR (in IP6), so based on a weak-strong simulation we calculated the diffusion as a function of the particles' action and used the result in the diffusion equation to find the evolution in time of the particles' distribution. This report is organized as follows: in section 2 we describe the beam parameters used in the simulation, in section 3 the modification in SixTrack which enables us to calculated diffusion more efficiently, in section 4 there is a description of the theory used in the lifetime calculation, in section 5 we compare data and simulation and finally in section 6 the show some dicussions and conclusions.

\section{Beam parameters}

For the RHIC pp run, the two proton beams collide at IP6 and IP8. The proton beam in the Blue ring circulates clockwise, while the proton beam in the Yellow ring circulates counter-clockwise. The proton beam parameters used in this study are show in Tab. 1. The linear chromaticities are corrected to $\xi_{x, y}=+2$ but we scanned this parameter using values between +2 to +0.5 and we also included in the lattice the multipole magnetic field errors in the triplet quadrupoles and separation dipole magnets in the IRs. The bunch intensity is varied between $N_{p}=1.0 \times 10^{11}$ and $N_{p}=2.0 \times 10^{11}$ and we assume that the proton beam has a Gaussian profile with a fixed width and no momentum spread. 
Table 1: Parameters for the proton beams

\begin{tabular}{lcc}
\hline \hline quantity & unit & value \\
\hline lattice & $\mathrm{m}$ & 3833.8451 \\
ring circumference & $\mathrm{GeV}$ & 100 \\
energy & - & 106.8 \\
relativistic $\gamma$ & - & $\mathrm{IP} 6, \mathrm{IP} 8$ \\
beam-beam collision points & $\mathrm{m}$ & 1 \\
$\beta_{x, y}^{*}$ at IP6 and IP8 & $\mathrm{m}$ & 10 \\
$\beta_{x, y}^{e}$ at IP10 & $\mathrm{m}$ & 10 \\
$\beta_{x, y}^{*}$ at all other IPs & - & $0.5-1.5 \times 10^{11}$ \\
proton beam & $\mathrm{mm} \cdot \mathrm{mrad}$ & 2.5 \\
particles per bunch $N_{p}$ & $\mathrm{~mm}$ & 0.146 \\
normalized transverse rms emittance $\epsilon_{x, y}$ & - & $(28.695,29.685) /(28.685,29.695)$ \\
transverse rms beam size at collision points $\sigma_{x, y}^{*}$ & - & $(2,2)$ \\
transverse tunes $\left(Q_{x}, Q_{y}\right)$ & - & $0.005-0.01$ \\
chromaticities $\left(\xi_{x}, \xi_{y}\right)$ & & \\
beam-beam parameter per IP $\xi_{\mathrm{p} \rightarrow \mathrm{p}}$ &
\end{tabular}

\section{Simulation Code}

In our study, the 6-D simplectic tracking code SixTrack [1] is used. In this code, the linear elements are treated as thick elements while the nonlinear elements are treated as thin-lenses. The beam-beam interaction calculation is based on a weak-strong model. SixTrack has an internal limitation on the particle number and in order to be able to simulate with an arbitrary number of particles at a time the source code was modified so a distribution file can be used as the initial particles coordinate input. This feature is achieved by applying a DO loop of the 64 pack which extended the maximum number of particles to 20000 and to activate this special simulation condition the lhe flag in the BEAM-BEAM element has to be set to 9. In order to be able to calculate the diffusion coefficient another DO loop was added in which the action of each particle is calculated and accumulated for 100 turns and then the averaged. A new output file is now created in which the rms spread of the action of each set of 64 particles is printed every 100 turns.

\section{Lifetime Calculation}

In order to estimate the beam lifetime, first it is necessary to calculate the particles' diffusion as a function of the initial amplitude. We used SixTrack to calculate the trajectories of 64 particles with the same initial action for $10^{5}$ turns and for each turn we calculate the rms spread $\left(\sigma_{J}\right)$ of the action for all the particles. We then average the rms action values over 100 turns to eliminate the short-term fluctuations due to phase-space orbit deviations from the linear model [2] that can be caused by weak resonances. 


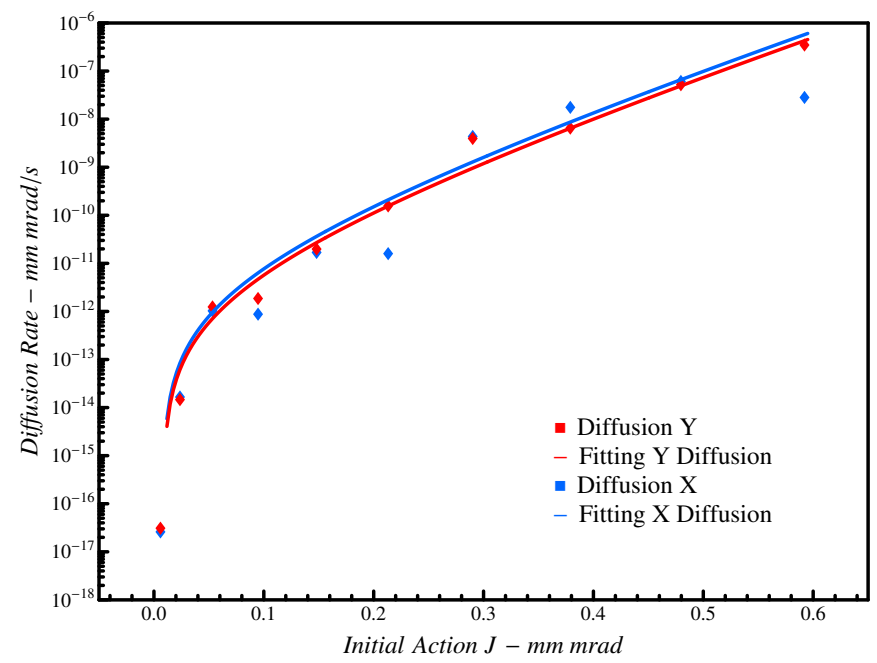

Figure 1: Example of the diffusion simulation results and the fitting for the working point above the diagonal and design tunes $(0.695,0.685)$.

The diffusion coefficient is then defined as

$$
D(J)=\lim _{N \rightarrow \infty} \frac{\langle J(N)-J(0)\rangle^{2}}{N}=\lim _{N \rightarrow \infty} \frac{\sigma_{J}^{2}(N)}{N}
$$

and in order to calculate its value we fit a line $\left(\sigma_{J}^{2}(N)=a+b N\right)$ over the rms action values for 1000 points spaced by 100 turns, so that we have $D(J)=b$. Once the diffusion points are calculated we fit the result using the expression $D(J)=A J+B J^{2} \exp (C J)$. This fitting function behaves like a parabola for the inner-most particles in the bunch which is in agreement with T. Sen [3] calculation for diffusion due to beam-beam effects and for the tails it has an exponential behavior to account for diffusion due to the lattice non-linearities. Some results for the diffusion simulation and the fitting are in Figure 1 for the working point above the diagonal.

From the diffusion simulation it is possible to calculate the diffusion coefficient for the $\mathrm{x}$ and $\mathrm{y}$ directions. Assuming that the diffusion coefficients are independent in both planes, i. e., we do not have any crossed terms, we can define a diffusion matrix with only diagonal elements

$$
\mathcal{D}=\left(\begin{array}{cc}
D_{x x} & 0 \\
0 & D_{y y}
\end{array}\right)
$$

Assuming furthermore that the diffusion in action is a Markov process and that the drift coefficient is half of the derivative of the diffusion coefficient (this is true for most Hamiltonian systems), we can describe the evolution of the phase-space distribution $\rho(J, t)$ 
by a diffusion equation

$$
\frac{\partial \rho\left(J_{x}, J_{y}, t\right)}{\partial t}=\frac{1}{2} \vec{\nabla} \cdot\left[\mathcal{D} \vec{\nabla} \rho\left(J_{x}, J_{y}, t\right)\right]
$$

and using that $\rho\left(J_{x}, J_{y}, t\right)=\rho_{x}\left(J_{x}, t\right) \rho_{y}\left(J_{y}, t\right)$ it is possible to separate equation 3 into two diffusion equations, one for each plane

$$
\frac{\partial \rho_{x, y}}{\partial t}=\frac{1}{2} \frac{\partial}{\partial J_{x, y}}\left(D_{x x, y y} \frac{\partial \rho_{x, y}}{\partial J_{x, y}}\right)
$$

assuming also that the machine has a physical aperture limitation at $J_{a}=a^{2} / \beta$ (primary collimator position, for example) and changing variables to $Z=J_{x, y} / J_{a}$ and $D^{\prime}=D /\left(2 J_{a}^{2}\right)$ we have that

$$
\frac{\partial f}{\partial t}=\frac{\partial}{\partial Z}\left(D^{\prime} \frac{\partial f}{\partial Z}\right)
$$

where $f(t, Z)$ is the new distribution function in the coordinate system and obeys the following boundary conditions

$$
f(Z, 0)=\frac{a^{2}}{2 \sigma^{2}} e^{-\left(a^{2} / 2 \sigma^{2}\right) Z} \quad \text { and } \quad f(1, t)=0
$$

In order to calculate the beam lifetime, or particle decay rate, it is necessary only to integrate the particle density for all values of the action, so

$$
N(t)=\int_{0}^{J_{a}} \rho\left(J_{x}, J_{y}, t\right) d J_{x} d J_{y}=\int_{0}^{J_{a}} \rho_{x}\left(J_{x}, t\right) d J_{x} \int_{0}^{J_{a}} \rho_{y}\left(J_{y}, t\right) d J_{y}
$$

Another point that should be taken into account in the calculation of lifetime is that the head-on beam-beam parameter varies with intensity and so the diffusion coefficient. The head-on effect accounts for the most part of the diffusion specially close to the beam core. In this case we have to recalculate $N(t)$ taking into account it's integrated value in the previous step and change the diffusion function accordingly. In order not to create artificial steps in the lifetime curve we had to choose a time interval for the integration of the diffusion equation $(N(t-\Delta t))$ in which the changes in intensity were small and also with $\Delta t$ sufficiently small compared to the store length (about 5 hours). For the cases simulated we used $\Delta t=18$ seconds and we simulated 3 intensity cases: $1,1.5$ and $2.0 \times 10^{11}$ protons/bunch and interpolated the diffusion coefficient for the intermediate intensities.

In order to benchmark the code for the lifetime calculation we did a series of simulations with different bunch intensities and the lattice from the 2006 proton run. Since in SixTrack the only effects that cause diffusion are magnet non-linearities and beambeam we calculated the beam-gas interaction component and added it to the diffusion coefficient. From the previous section the diffusion fitted to the simulation points was $D(J)=A J+B J^{2} e^{C J}$ the extra term due to gas scattering is linear with the action and so $D_{\text {gas }}=K J$, with $K=2 / \gamma\left(d \varepsilon_{N} / d t\right)_{\text {gas }}=6 \times 10^{-12} \mathrm{~mm} \mathrm{mrad} / \mathrm{s}$. 


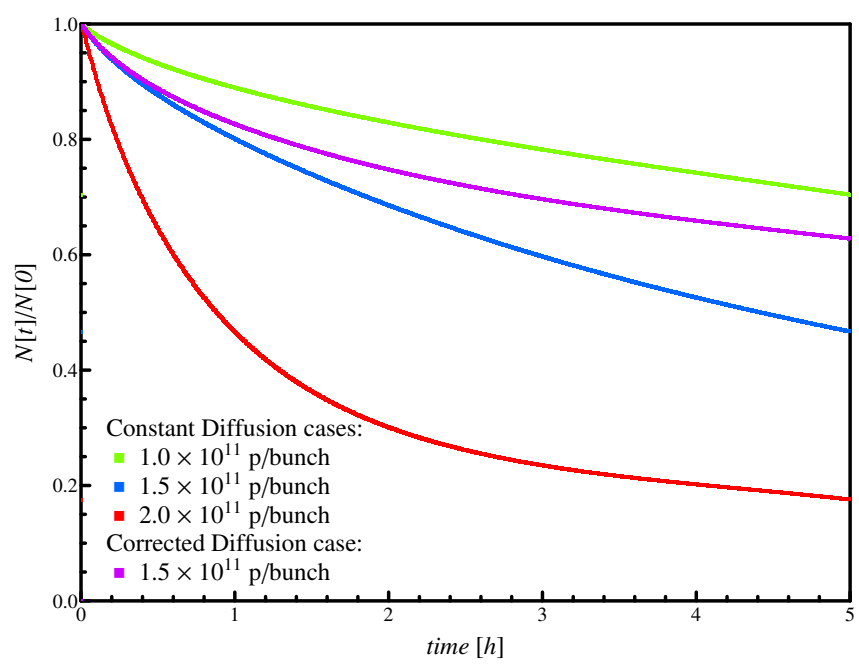

Figure 2: Example of the lifetime calculation results. The three lines (green, blue and red) represent the lifetime calculated using a constant diffusion coefficient, which overestimates the decay rate. The purple line is a calculation for the case with $1.5 \times 10^{11}$ protons/bunch, that is equivalent to the blue line in the plot, but with the correction on the diffusion coefficient as the intensity decreases.

\section{Results}

In order to test the lifetime calculation we compared the simulation results with physics stores from the 2006 and 2008 polarized proton runs. The results and discussions were separated into two general cases: low and high bunch intensity stores. This separation was necessary since as the run progresses and the total bunch charge is increased some of the machine parameters are optimized in order to keep the beam lifetime and the experiments background acceptable. There are also some assumptions made when developing the theory that have to be taken into account when comparing the simulation results with experiments. First the initial emittance of the blue and yellow rings should be as close as possible as well as the initial intensity. This comes from the fact that we simulate just one beam, the weak one, and when solving the diffusion equation assumes that the other beam intensity decays at the same rate so that we just need to adjust the beam-beam parameter, i. e., the diffusion function accordingly. Second, the initial tunes should be close to the design ones so that we can compare simulation and data more accurately and finally the initial intensity data point is taken only after the collimators are already in, so that we have the absorbing boundary condition around $6 \sigma_{x, y}$ as assumed in the theory. We simulated the diffusion for 3 different values of the beam intensity: 1.0, 1.5 and $2.0 \times 10^{11}$ protons/bunch so that we are able to calculate the lifetime for any intensity in between those values. 
FIGURE A

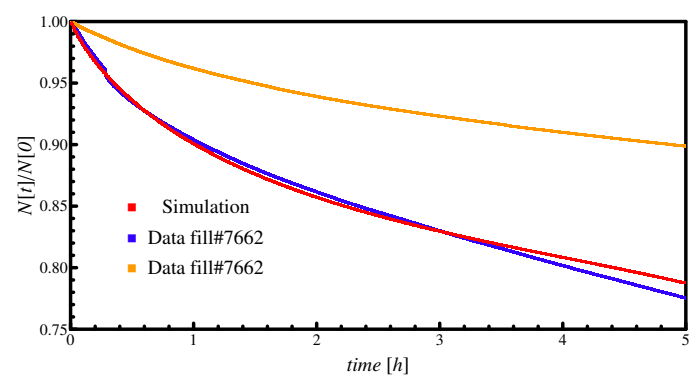

FIGURE C

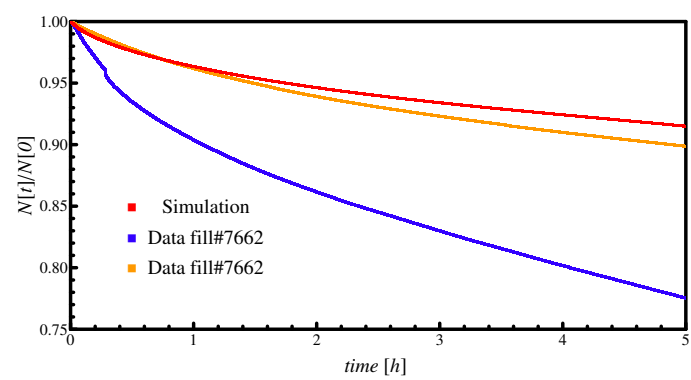

FIGURE B

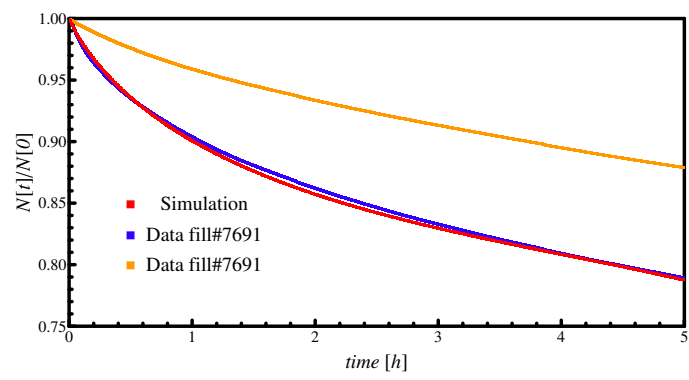

FIGURE D

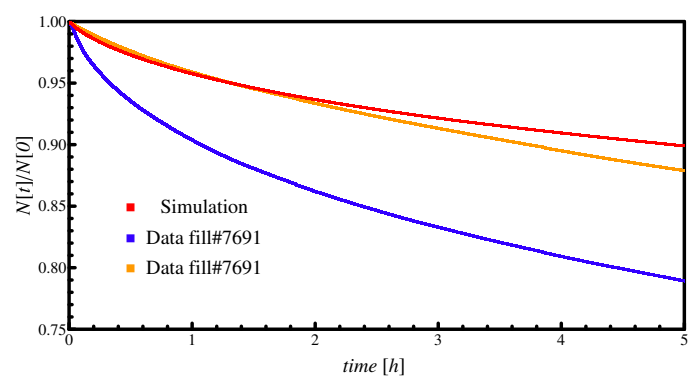

Figure 3: Example of the lifetime calculation results. In all cases the initial bunch intensity is around $1 \times 10^{11}$ protons and the chromaticity is +1 . The plots show the beam decay for the Yellow (yellow line) and Blue (blue line) beams and also the beam decay predicted from the diffusion simulation (red line). (A) and (B) are the cases with the fractional tune $(0.695,0.685)$ used in the simulation and (C) and (D) the factional tune in the simulation is $(0.685,0.695)$. The number displayed in each plot correspond to the filling number and the data is from the 2006 polarized proton run in which the blue beam was set at the working point below the diagonal and the yellow beam above.

\subsection{Case 1: Low Bunch Intensity Stores}

For the low intensity cases all the machines parameters are the same as the ones listed in Table 1. In Figure 3 are some cases for the 2006 polarized proton run and in Figure 4 For the 2008 run. For all low intensity cases changes in tunes of 0.002 and/or chromaticity of 1 unit in both planes do not represent a substantial change in the simulated diffusion coefficients. A comparison of the calculated and measured data are show in Table 2 for the beam decay after 3 hours and 5 hours into the store.

In the 2006 the blue ring working point was set below the diagonal - $(0.695,0.685)$ while the Yellow ring was above - $(0.685,0.695)$. Note that measured and the simulated normalized intensities agree whithin 0.02 or $2 \%$ in the worst case. For the 2008 run the agreement is not as good as 2006, in this run the working point in both rings was near $(0.695,0.685)$ where the dynamic aperture is larger, and the simulation deviates from the data by no more than $6 \%$ or a difference of 0.08 in $N(t) / N(0)$. 
FIGURE A

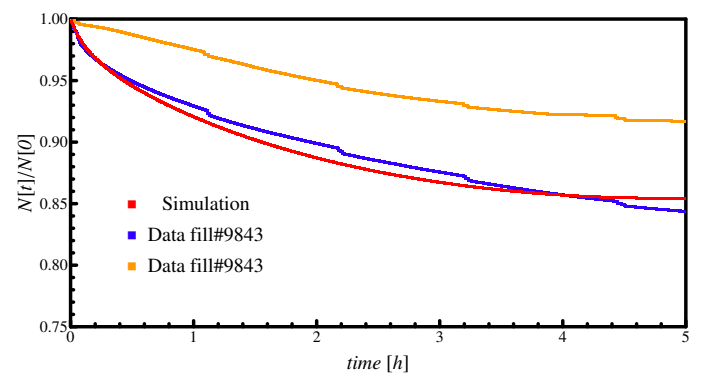

FIGURE B

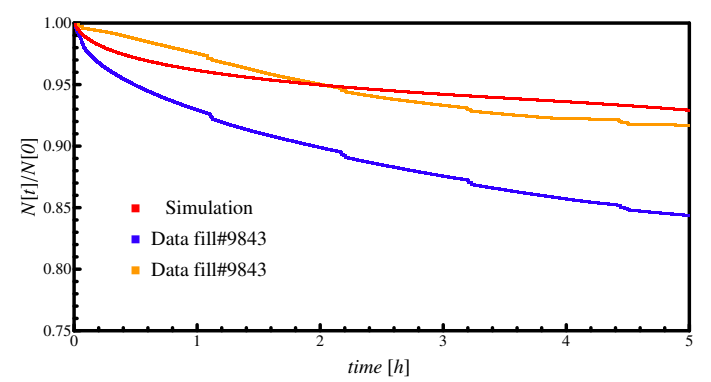

Figure 4: Example of the lifetime calculation results. In all cases the initial bunch intensity is around $0.7 \times 10^{11}$ protons and the chromaticity +2 . The plots show the beam decay for the Yellow (yellow line) and Blue (blue line) beams and also the beam decay predicted from the diffusion simulation (red line). (A) and (B) are both fractional tune $(0.695,0.685)$ used in the simulation and the only difference is the emittance of each beam. The number displayed in each plot correspond to the filling number and the data is from the 2008 polarized proton run in which both, the blue and yellow beam, were set at the working point below the diagonal.

Table 2: Comparison of the calculated lifetimes and data for the 2006 and 2008 proton runs. The beam decay is compared at the middle ( 3 hours) and at the end ( 5 hours) of the physics store.

\begin{tabular}{lcccc}
\hline \hline Store \# & \multicolumn{2}{c}{$N(t=3$ hours $) / N(0)$} & \multicolumn{2}{c}{$N(t=3$ hours $) / N(0)$} \\
data & simulation & data & simulation \\
\hline Blue Beam & & & & \\
7662 & 0.83 & 0.83 & 0.78 & 0.79 \\
7691 & 0.84 & 0.83 & 0.79 & 0.79 \\
9843 & 0.88 & 0.87 & 0.84 & 0.85 \\
7918 & 0.90 & 0.90 & 0.87 & 0.88 \\
7921 & 0.85 & 0.85 & 0.81 & 0.82 \\
9979 & 0.86 & 0.82 & 0.83 & 0.80 \\
9981 & 0.84 & 0.82 & 0.82 & 0.80 \\
Yellow Beam & & & & \\
7662 & 0.93 & 0.93 & 0.86 & 0.92 \\
7691 & 0.92 & 0.92 & 0.88 & 0.90 \\
9843 & 0.93 & 0.94 & 0.92 & 0.93 \\
7918 & 0.83 & 0.90 & 0.82 & 0.88 \\
7921 & 0.75 & 0.85 & 0.73 & 0.82 \\
9979 & 0.92 & 0.82 & 0.89 & 0.80 \\
9981 & 0.94 & 0.82 & 0.92 & 0.80 \\
\hline \hline
\end{tabular}




\subsection{Case 2: High Bunch Intensity Stores}

The behavior of the lifetime for high bunch intensity is quite different from the low intensity ones and the simulation results showed before do not match the data from both runs, 2006 and 2008. It is often observed that during the run, when the bunch intensity is increased, there is a tendency for shifting the tunes to higher values and shifting the chromaticity down to reduce the tune spread and improve lifetime. Since those changes are performed slightly different in each shift we cannot tell the final values of tunes and chromaticities. In order to get a better matching of simulation and data we calculated the diffusion with a tune change in both planes of +0.001 and +0.002 , we also changed the chromaticity and instead of +2 units used +1 and +0.5 units, keeping the tunes at the nominal values. We could not see any difference in diffusion from the tune changes and also for the case with $\xi_{x, y}=+1$ however there is a noticeable difference for the case with $\xi_{x, y}=+0.5$. In Figure 5 are some results for the last simulated scenario and the comparison with the data.

For the high intensity cases the agreement between simulations and experiments is only acceptable in the case of the Blue ring as show in Table 2, which is 0.04 or $4 \%$ in the worst case. We could no get a closer agreement for the Yellow ring for any of the scenarios simulated (different tunes and chromaticities) and even though the tune of both rings and the emittances during the 2008 run were close the behavior of the lifetime is quite different (vide Figure 5).

\section{Conclusions}

Using a modified version of SixTrack we are now able to calculate the action diffusion of a set of 64 particles. Using the diffusion results for different machine setups (different working points and chromaticities) we are able to predict the RHIC proton beam lifetime within $6 \%$ of accuracy in beam decay $(N(t) / N(0))$ after 5 hours at store. The prediction for the low intensity stores is very good for both rings (Yellow and Blue) and in both runs (2206 and 2008). For this cases small changes in tune and chromaticities do not have a great impact on the beam lifetime, which is observed experimentally. With a low intensity store the tune spread in the tune footprint due to beam-beam collisions is small and only weak resonances are crossed thus the weak dependence with tune and chromaticities. The high intensity cases are different and only the blue ring presents a predictable behavior when we use a chromaticity of +0.5 . It is important to remember that the measurement of chromaticity in RHIC is not performed very often therefore we do not have a direct comparison of data and simulation with close values of chromaticity, however as the intensity of the stored beam increases during a run there is the tendency of reducing the chromaticities of both beams to improve lifetime. The Yellow beam, even though with very close tunes and emittances if compared with the blue beam for the 2008 run, has a smaller diffusion rate and consequently a better lifetime and we could not reproduce its lifetime with small scans in tune and chromaticity. A further investigation of possible parameters which have a influence in the Yellow beam behavior is necessary. 
FIGURE A

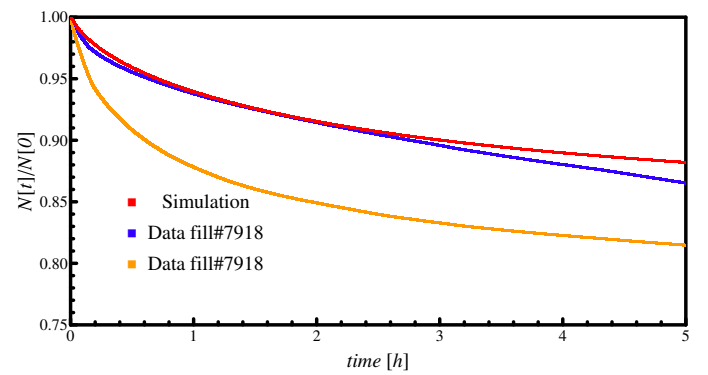

FIGURE C

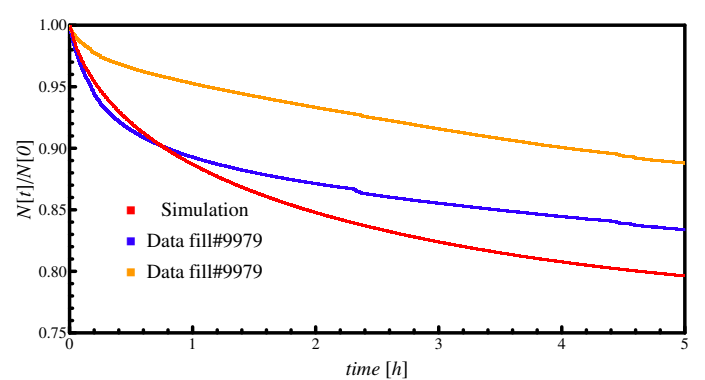

FIGURE B

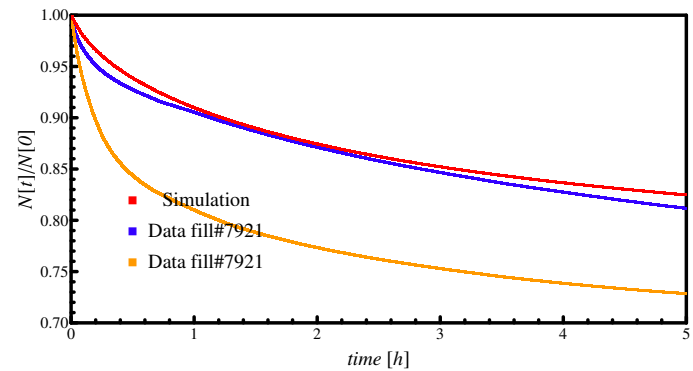

FIGURE D

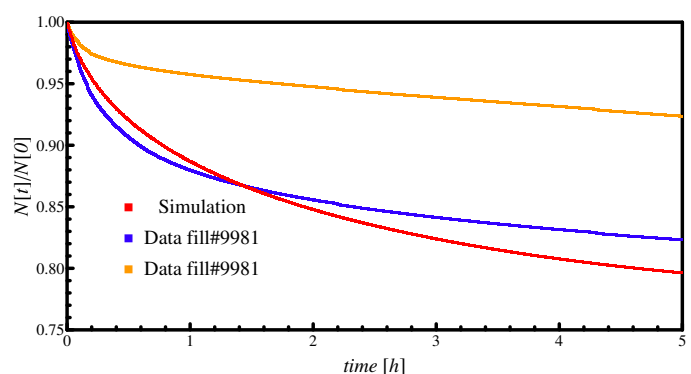

Figure 5: Example of the lifetime calculation results. In all cases the chromaticity is set at +0.5 and the tune is $(0.695,0.685)$. The plots show the beam decay for the Yellow (yellow line) and Blue (blue line) beams and also the beam decay predicted from the diffusion simulation (red line). (A) $N_{b}=1.3 \times 10^{11}$ protons/bunch, (B) $N_{b}=1.4 \times 10^{11}$ protons/bunch and (C) and (D) $N_{b}=1.6 \times 10^{11}$ protons $/$ bunch. The number displayed in each plot correspond to the filling number and the data is from the 2006 (A and $\mathrm{B}$ ) and 2008 (C and D) polarized proton run in which both, the blue and yellow beam, were set at the working point below the diagonal. 


\section{References}

[1] F. Schmidt, "SixTrack Manual", Version 4.1, CERN/SL/94-56 (AP). Availbale at http://frs.home.cern.ch/frs/.

[2] J. Irwin, "Diffusive losses from SSC particle bunches due to long-range beam-beam interactions", SSC-233, September 1989.

[3] T. Sen, J. A. Ellison, Phys. Rev. Lett. vol. 77, number 6 page 1051. 